\title{
Trends in Troponin-Only Testing for AMI in Academic Teaching Hospitals and the Impact of Choosing Wisely ${ }^{\circledR}$
}

\author{
Micah T. Prochaska, MD, MS ${ }^{1 \star}$, Samuel F. Hohmann, PhD, MS-HSM²,3, Matthew Modes, MD, MPH${ }^{4}$, Vineet M. Arora, MD, MAPP1 \\ ${ }^{1}$ Department of Medicine, The University of Chicago, Chicago, Illinois; '2Vizient, Chicago, Illinois; ${ }^{3}$ Department of Health Systems Management, Rush \\ University, Chicago, Illinois; ${ }^{4}$ Department of Medicine, University of Washington, Seattle, Washington.
}

\begin{abstract}
BACKGROUND: Identifying hospitals that are both early and consistent adopters of high-value care can help shed light on the culture and practices at those institutions that are necessary to promote high-value care nationwide. The use of troponin to diagnose acute myocardial infarction (AMI), and not to test for myoglobin or creatine kinase-MB (CK-MB), is a high-value recommendation of the Choosing Wisely ${ }^{\circledR}$ campaign.
\end{abstract}

OBJECTIVE: To examine the variation in cardiac biomarker testing and the effect of the Choosing Wisely ${ }^{\circledR}$ troponin-only recommendation for the diagnosis of AMI.

DESIGN: A retrospective observational study using administrative ordering data from Vizient's Clinical Database/Resource Manager.

SETTING: Ninety-one academic medical centers from the fourth quarter of 2013 through the third quarter of 2016.

PATIENTS: Hospitalized patients with a principal discharge diagnosis of AMI.
INTERVENTION: The Choosing Wisely ${ }^{\circledR}$ recommendation to order troponin-only testing to diagnose AMI was released during the first quarter of 2015.

RESULTS: In 19 hospitals, troponin-only testing was consistently ordered to diagnose AMI before the Choosing Wisely ${ }^{\circledR}$ recommendation and throughout the study period. In 34 hospitals, both troponin and myoglobin/CK-MB were ordered to diagnose AMl even after the Choosing Wisely ${ }^{\circledR}$ recommendation. In 26 hospitals with low rates of troponin-only testing before the Choosing Wisely ${ }^{\circledR}$ recommendation, the release of the recommendation was associated with a statistically significant increase in the rate of troponin-only testing to diagnose AMI.

CONCLUSION: In institutions with low rates of troponin-only testing prior to the Choosing Wisely ${ }^{\circledR}$ recommendation, the recommendation was associated with a significant increase in the rate of troponin-only testing. Journal of Hospital Medicine 2017;12:957-962. Published online first September 20, 2017. (C) 2017 Society of Hospital Medicine
Evidence suggests that troponin-only testing is the superior strategy to diagnose acute myocardial infarction (AMI). ${ }^{1}$ Because of this, in February 2015, the Choosing Wisely ${ }^{\circledR}$ campaign issued a recommendation to use troponin I or $\mathrm{T}$ to diagnose AMI, and not to test for myoglobin or creatine kinase-MB $(\mathrm{CK}-\mathrm{MB}) .^{2}$ This recommendation was in line with guidelines from the American Heart Association and the American College of Cardiology, which recommended that myoglobin and CK-MB are not useful and offer no benefit for the diagnosis of acute coronary syndrome. ${ }^{3}$ Some institutions have developed interventions to promote troponin-only testing, reporting substantial cost savings and no negative consequences. ${ }^{4,5}$

Despite these successes, it is likely that institutions vary with respect to the adoption of the Choosing Wisely ${ }^{\circledR}$ troponin-only testing recommendation. ${ }^{6}$ Implementing this recommendation requires both promoting clinician behav-

\footnotetext{
*Address for correspondence and reprint requests: Micah T. Prochaska, MD, MS, University of Chicago, 5841 S. Maryland Avenue, MC 5000. Chicago, IL 60637; Telephone: 773-702-6988; Fax: 773-795-7398; E-mail: mprochas@ medicine.bsd.uchicago.edu
}

Additional Supporting Information may be found in the online version of this article.

Received: November 29, 2016; Revised: May 5, 2017;

Accepted: May 21, 2017

2017 Society of Hospital Medicine DOI 10.12788/jhm.2846 ior change and a strong institutional culture of high-value care. ${ }^{7}$ Understanding the variation across institutions of troponin-only testing could inform how to promote high-value care recommendations nationwide. We aimed to describe patterns of troponin, myoglobin, and CK-MB testing in a sample of academic teaching hospitals before and after the Choosing Wisely ${ }^{\circledR}$ recommendation.

\section{METHODS}

Troponin, myoglobin, and CK-MB ordering data were extracted from Vizient's (formerly University HealthSystem Consortium, Chicago, IL) Clinical Database/Resource Manager $\left(\mathrm{CDB} / \mathrm{RM}^{\circledR}\right)$ for all patients with a principal discharge diagnosis of AMI at all hospitals reporting all 36 months from the fourth quarter of 2013 through the third quarter of 2016. This period includes time both before and after the Choosing Wisely ${ }^{\circledR}$ recommendation, which was released in the first quarter of 2015. Vizient's CDB/RM contains ordering data for 300 academic medical centers and their affiliated hospitals and includes the discharge diagnoses for patients cared for by these institutions. Only patients with a principal discharge diagnosis of AMI were included because the Choosing Wisely ${ }^{\circledR}$ recommendation is specific with regard to troponin-only testing for the diagnosis of AMI. Patients with a principal diagnosis code for subcategories of myocardial ischemia (eg, stable angina, unstable angina) were 
not included because of the large number of diagnosis codes for these subcategories (more than 100 in the International Classification of Diseases, Ninth Revision and the International Classification of Diseases, Tenth Revision) and because the variation in their use across institutions within the dataset limited the utility of using these codes to consistently and accurately identify patients with myocardial ischemia. Moreover, the diagnosis of AMI encompasses the subcategories of myocardial ischemia. ${ }^{8}$

Hospital rates of ordering cardiac biomarkers (troponin-only or troponin and myoglobin/CK-MB) were determined overall for the entire study period and for each quarter of the study period based on the total patients with a discharge diagnosis of AMI. For each quarter of the 12 study quarters, all the hospitals were divided into tertiles based on their rate of troponin-only testing per discharge diagnosis of AMI. Hospitals were then classified into 3 groups based on their tertile ranking over the full 12 study quarters. The first group included hospitals whose rate of troponin-only testing placed them in the top tertile for each and all quarters throughout the study period. The second group included hospitals whose troponin-only testing rate placed them in the bottom tertile for each and all quarters throughout the study period. The third group included hospitals whose troponin-only testing rate each quarter led to either an increase or decrease in their tertile ranking throughout the study period. $\chi^{2}$ tests were used to test for bivariate associations among hospitals based on their rate of troponin-only testing and hospital size (number of beds), their regional geographic location, the volume of AMI patients seen at the hospital, whether the primary physician during the hospitalization was a cardiologist or other provider, and the hospitals' quality ratings. Quality rating was based on an internal Vizient rating and the "Best Hospitals for Cardiology and Heart Surgery Rankings" as published in the US News $\mathcal{E}$ World Report. ${ }^{9}$ The Vizient quality rating is based on a composite score that combines scores from the domains of quality (hospital quality incentive scores), safety (patient safety indicators), patient-centeredness (Hospital Consumer Assessment of Healthcare Providers and Systems Hospital Survey), and equity (distribution of care by race/ethnicity, gender, and age). Simple slopes were calculated to determine the rate of change in troponin-only testing for each study quarter, and Student $t$ tests were used to compare the rates of change of these simple slopes across study quarters.

\section{RESULTS}

Of the 300 hospitals in Vizient's CDB/RM, 91 (30\%, 91/300) had full reporting of data throughout the study period. These hospitals had a total of 106,954 inpatient discharges with a principal diagnosis of AMI during the study period. The overall rates of troponin-only testing for AMI discharges by hospital varied from $0 \%$ to $87.4 \%$ (Figure 1). The mean rate of troponin-only testing across all patients with a discharge diagnosis of AMI was $29.2 \%$ at the start of the study (fourth quarter of 2013) and $53.5 \%$ at the end of the study (third quarter 2016; Supplemental Figure). Nineteen hospitals (21\%, 19/91; 27,973 discharges) had high rates of troponin-only testing for AMI and were in the top tertile of all hospitals throughout the study period. Thirty-four hospitals (37\%, 34/91; 35,080 discharges) ordered both troponin and myoglobin/CK-MB tests to diagnose AMI, and they were in the bottom tertile of all hospitals throughout the study period. In the 38 hospitals (42\%, 38/91; 43,090 discharges) that were not in the top or bottom tertile for all study quarters, the rate of troponin-only testing for AMI increased at each hospital during each quarter of the study period (Table).

\section{Pattern of Troponin-Only Testing by Hospital Size}

Of the hospitals in the top tertile of troponin-only testing throughout the study period, the majority had $\geq 500$ beds (13/19), but the highest rate of troponin-only testing was in hospitals that had $<250$ beds ( $\mathrm{n}=4$, troponin-only testing rate of $82 / 100$ patients). Additionally, in hospitals that improved their troponin-only testing during the study period, hospitals that had $<500$ beds had higher rates of troponin-only testing than did hospitals with $\geq 500$ beds. The differences in the rates of troponin-only testing across the 3 groups of hospitals and hospital size were statistically significant $(P<0.0001$; Table).

\section{Pattern of Troponin-Only Testing by Geographic Region}

The rate of troponin-only testing also varied and was statistically significantly different when comparing the 3 groups of hospitals across geographic regions of the country $(P<0.0001)$. Of the hospitals in the top tertile of troponin-only testing throughout the study period, the majority were in the Midwest $(n=6)$ and Mid-Atlantic $(n=5)$ regions. However, the rate of troponin-only testing for AMI in this group was highest in hospitals in the West (86/100 patients) and/or Southeast (75/100 patients) regions, although this rate was based on a small number of hospitals in these geographic areas ( $n=1$ in the West, $n=2$ in the Southeast). Of hospitals in the bottom tertile of troponin-only testing throughout the study period, the majority were in the Mid-Atlantic region $(n=10)$. Hospitals that increased their troponin-only testing during the study period were predominantly in the Midwest $(\mathrm{n}=12)$ and Mid-Atlantic regions ( $\mathrm{n}$ $=11$; Table), with the hospitals in the Midwest having the highest rate of troponin-only testing in this group.

\section{Pattern of Troponin-Only Testing by Volume of AMI Patients}

Of the hospitals in the top tertile of troponin-only testing during the study period, the majority cared for $\geq 1500$ AMI patients $(n=9)$, but interestingly, among these hospitals, those caring for a smaller volume of AMI patients all had higher rates of troponin-only testing per 100 patients $(P<0.0001$; Table $)$. There was no other obvious pattern of troponin-only testing based on the volume of AMI patients cared for in hospitals in either the bottom tertile of troponin-only testing or hospitals that improved troponin-only testing during the study period. 
TABLE. Hospital Characteristics and Bivariate Associations by Rate of Troponin-Only Testing

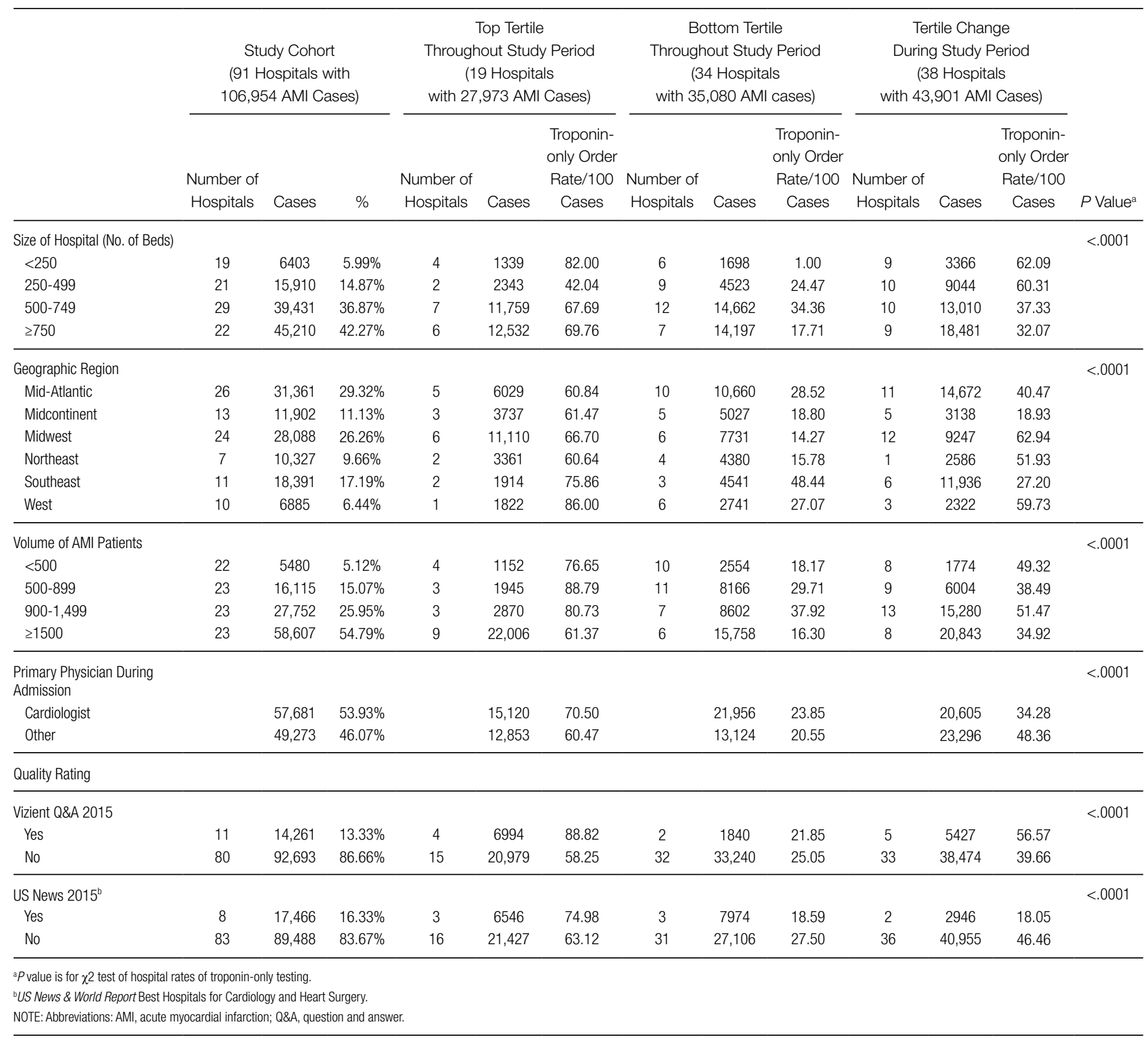

\section{Pattern of Troponin-Only Testing by Physician Type}

Of the hospitals in the top tertile of troponin-only testing throughout the study period, those where a cardiologist cared for patients with AMI had higher rates of troponin-only testing (71/100 patients) than did hospitals where patients were cared for by a noncardiologist (60/100 patients). However, of the hospitals that improved their troponin-only testing during the study period, higher rates of troponin-only testing were seen in hospitals where patients were cared for by a noncardiologist (48/100 patients) compared with patients cared for by a cardiologist (34/100 patients; Table). These differences in hospital rates of troponin-only testing during the study period based on physician type were statistically significant $(P<0.0001$; Table $)$.

\section{Pattern of Troponin-Only Testing by Quality Rating}

Hospitals that were in the top tertile of troponin-only testing and were rated highly by Vizient's quality rating or recognized as a top hospital by the US News $\mathcal{E}$ World Report had higher rates of troponin-only testing per 100 patients than did hospitals in the top tertile that were not ranked highly by Vizient's quality rating or recognized as a top hospital by the US News $\mathcal{E}$ World Report. However, the majority of hospitals in the top tertile of troponin-only testing were not rated highly by Vizient $(\mathrm{n}=15)$ or recognized as a top hospital by the US News $\mathcal{E}$ World Report $(\mathrm{n}=16)$. The large majority of hospitals in the bottom tertile of troponin-only testing were not recognized as high-quality hospitals by Vizient $(\mathrm{n}=32)$ or the US News $\mathcal{E}$ World Report $(\mathrm{n}=31)$. 


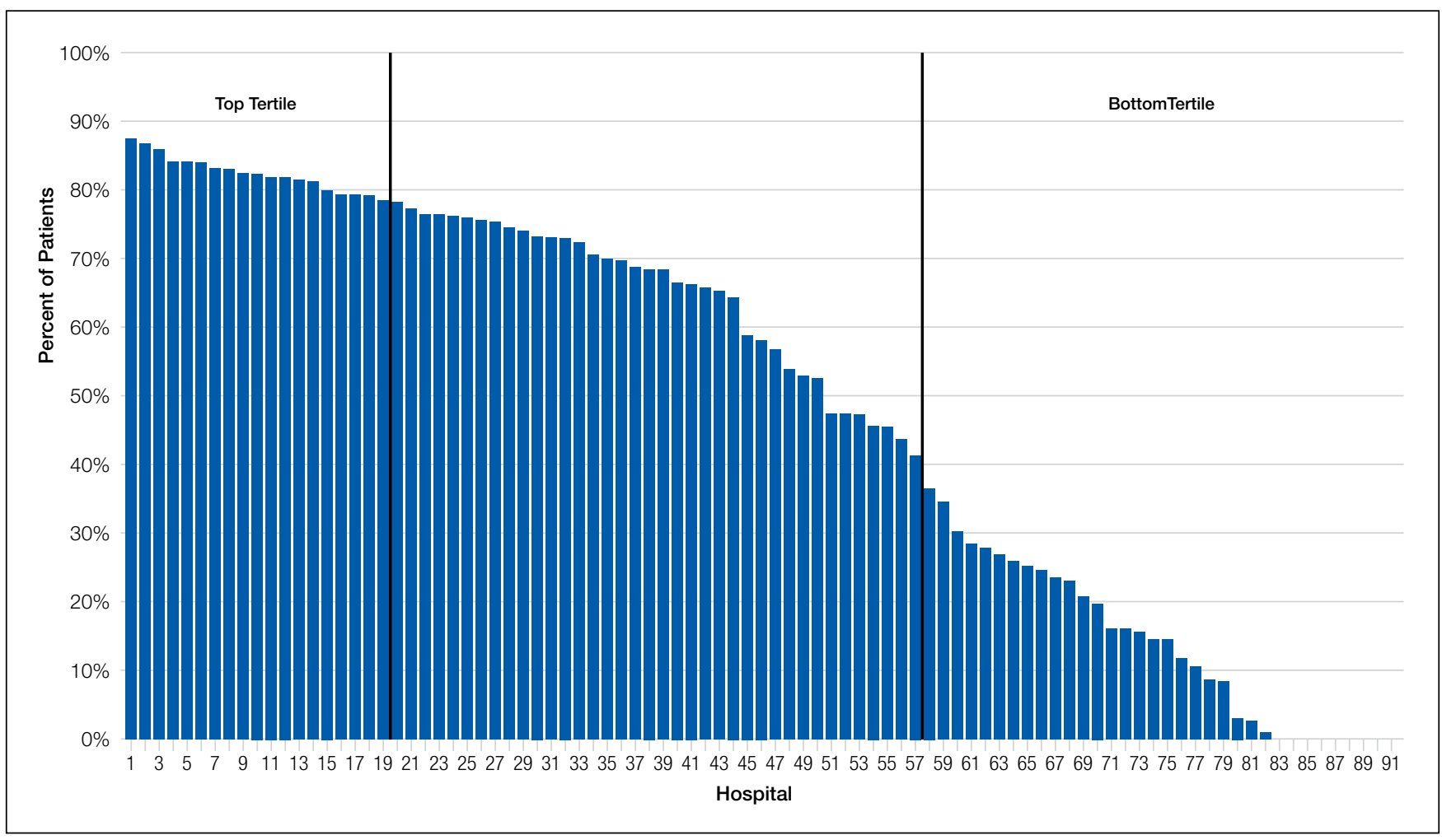

FIG 1. The proportion of troponin-only testing for the diagnosis of AMI by hospitals across the study period. NOTE: Abbreviation: AMI, acute myocardial infarction.

Of the hospitals that improved their troponin-only testing during the study period, the majority were not recognized as high-quality hospitals by Vizient $(\mathrm{n}=33$ ) or the US News $\mathcal{E}$ World Report $(\mathrm{n}=36)$, but among this group, those hospitals recognized by Vizient as high quality $(n=5)$ had the highest rate of troponin-only testing (57/100 patients). The differences in the rate of troponin-only testing across the different groups of hospitals and quality ratings were statistically significant $(P<0.0001$; Table $)$.

\section{The Effect of Choosing Wisely ${ }^{\circledR}$ on Troponin-Only Testing}

While in many institutions the rates of troponin-only testing were increasing before the Choosing Wisely ${ }^{\circledR}$ recommendation was released in 2015, the release of the recommendation was associated with a significant increase in the rate of troponin-only testing in the institutions that were in the bottom tertile of troponin-only testing prior to the release of the recommendation but moved to the top tertile after the release of the recommendation $(n=5)$. The slope percentage of the rate of change of the 5 hospitals that went from the bottom tertile to the top tertile after the release of the Choosing Wisely ${ }^{\circledR}$ recommendation was $5.7 \%$. Additionally, the Choosing Wisely ${ }^{\circledR}$ recommendation was associated with an accelerated rate of troponin-only testing in hospitals moving from the bottom tertile before the release of the recommendation to the middle tertile after the recommendation $(\mathrm{n}=15$; slope $=3.2 \%)$ and in hospitals moving from the middle tertile before the release of the recommendation to the top tertile after $(n=6$; slope $=2.4 \%)$
(Figure 2). For all of these hospitals $(n=26)$, the increased rate of troponin-only testing in the study quarter after the Choosing Wisely ${ }^{\circledR}$ recommendation was statistically significantly higher and different from the rate of troponin-only testing in all other study quarters, except for the period between 2014 quarter 3 and quarter $4(P=0.08)$, the period between 2015 quarter 2 and quarter $3(P=0.18)$, and 2015 quarter 3 and quarter $4(P=0.06)$, where the effect did not quite reach statistical significance (Figure 3 ).

\section{DISCUSSION}

In a broad sample of academic teaching hospitals, there was an overall increase in the rate of troponin-only testing starting from the fourth quarter of 2013 through the third quarter of 2016. However, there was wide variation in the adoption of troponin-only testing for AMI across institutions. Our study identified several high-performing hospitals where the rate of troponin-only testing was high prior to and after the Choosing Wisely ${ }^{\circledR}$ troponin-only recommendation. Additionally, we identified several poor-performing hospitals, which even after the release of the Choosing Wisely ${ }^{\circledR}$ recommendation continue to order both troponin and myoglobin/CK-MB tests for the diagnosis of AMI. Lastly, we identified several hospitals in which the release of the Choosing Wisely® recommendation was associated with a significant increase in the rate of troponin-only testing for the diagnosis of AMI. The high-performing hospitals in our sample that were in the top tertile of troponin-only testing throughout the study period are "early adopters," having already institut- 


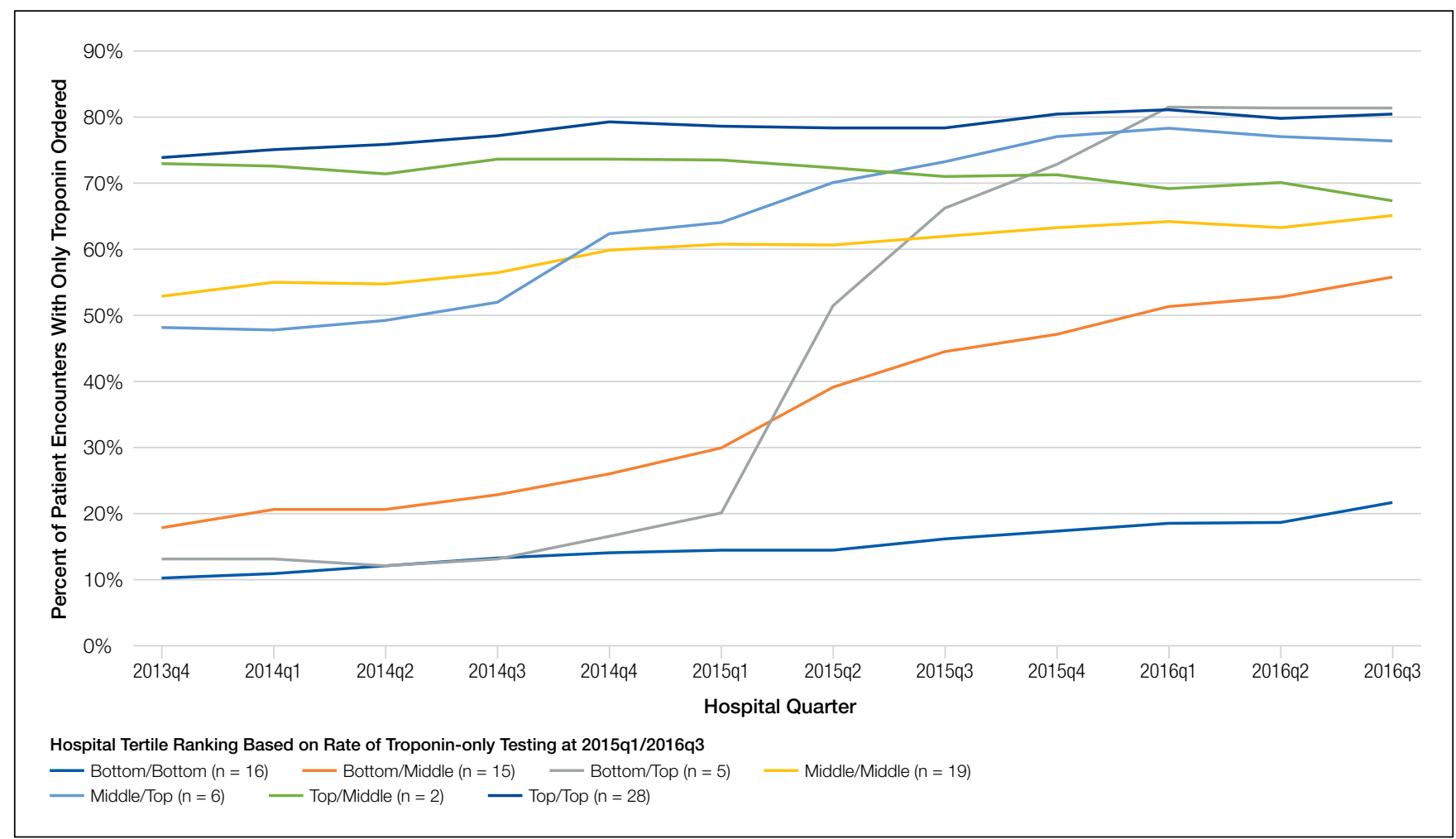

FIG 2. Hospital percentage of patient encounters and tertile ranking of troponin-only testing to diagnose AMI by study quarter. NOTE: Abbreviation: AMI, acute myocardial infarction.

ed troponin-only testing before the release of the Choosing Wisely ${ }^{\circledR}$ troponin-only recommendation. These hospitals vary in size, geographic region of the country, volume of AMI patients cared for, whether AMI patients are cared for by a cardiologist or other provider, and quality rating. Interestingly, in these hospitals, AMI patients admitted under the care of a cardiologist had higher rates of troponin-only testing than when admitted under another physician type. This is perhaps not surprising given that cardiologists would be the most likely to be aware of the data supporting troponin-only testing prior to the Choosing Wisely ${ }^{\circledR}$ recommendation and the most likely to institute interventions to promote troponin-only testing and disseminate this knowledge across their institution. These institutions and their practice of troponin-only testing before the Choosing Wisely ${ }^{\circledR}$ recommendation represent the idea of positive deviance, ${ }^{10}$ whereby they had identified troponin-only testing as a superior strategy and instituted successful initiatives to reduce the use of unnecessary myoglobin and CK-MB testing before their peer hospitals and the release of the Choosing Wisely ${ }^{\circledR}$ recommendation. Further efforts to explore and understand the additional factors that define the hospitals that had high rates of troponin-only testing prior to the Choosing Wise$1 y^{\circledR}$ recommendation may be helpful to understanding the necessary culture and institutional factors that can promote high-value care.

In the hospitals that demonstrated increasing adoption of troponin-only testing, there are several interesting pat- terns. First, among these hospitals, smaller hospitals tended to have higher overall rates of troponin-only testing per 100 patients than larger hospitals. Additionally, the hospitals with the highest rates were located in the Midwest region. These hospitals may be learning from and following the high-performing institutions observed in our data that are also located in the Midwest. Additionally, among the hospitals that significantly increased their rate of troponin-only testing, we see that the Choosing Wisely ${ }^{\circledR}$ recommendation appeared to facilitate accelerated adoption of troponin-only testing. In these institutions, it is likely that the impact of Choosing Wisely ${ }^{\circledR}$ was significant because there was attention to high-value care and already an existing movement underway to institute such high-value practices. For example, natural champions, leadership, infrastructure, and a supportive culture may all be prerequisites for Choosing Wise$1 y^{\circledR}$ recommendations to become institutionally adopted.

Lastly, in the hospitals that have continued to order myoglobin and CK-MB, future work is needed to understand and overcome barriers to adopting high-value care practices.

There are several limitations to this study. First, because this was an observational study, we cannot prove a causal relationship between the Choosing Wisely ${ }^{\circledR}$ recommendation and the increased rates of troponin-only testing. Additionally, the Vizient CDB/RM contains reporting data for a limited number of academic medical centers only, and therefore, these results may not represent practices at nonacademic or even other academic medical centers. Our study 


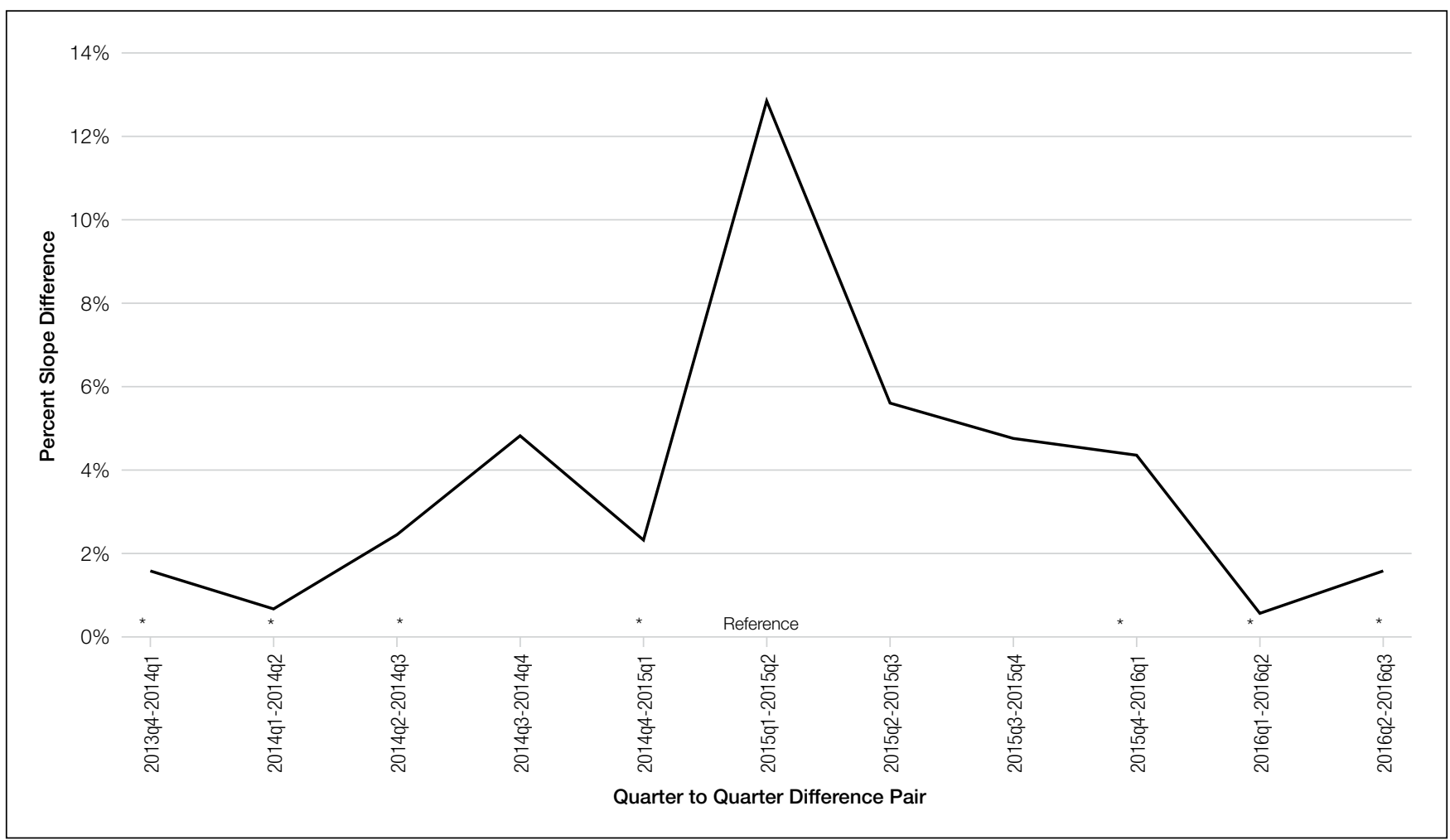

FIG 3. Average quarterly rate of change in troponin-only testing among hospitals that improved their troponin-only testing tertile.

NOTE: ${ }^{*}<.05$

only included patients with a principal discharge diagnosis of AMI because the Choosing Wisely ${ }^{\circledR}$ recommendation to order troponin-only is specific for diagnosing patients with AMI. However, it is possible that the Choosing Wisely ${ }^{\mathbb{B}}$ recommendation also has affected provider ordering in patients with diagnoses such as chest pain or angina, and these affects would not be captured in our study. Lastly, because instituting high-value care practices take time, our follow-up time may not have been long enough to capture improvement in troponin-only testing at institutions responding to and attempting to adhere to the Choosing Wisely ${ }^{\circledR}$ rec- ommendation to order troponin-only testing for patients with AMI.

Disclosure: No other individuals besides the authors contributed to this work. This project was not funded or supported by any external grant or agency. Dr. Prochaska's institute received funding from the Agency for Research Healthcare and Quality for a K12 Career Development Grant (AHRQ K12 HS023007) outside the submitted work. Dr. Hohmann and Dr Modes have nothing to disclose. Dr. Arora receives financial compensation as a member of the Board of Directors for the American Board of Internal Medicine and has received grant funding from the ABIM Foundation. She also receives royalties from McGraw Hill.

\section{References}

1. Pickering JW, Than MP, Cullen L, et al. Rapid rule-out of acute myocardial infarction with a single high-sensitivity cardiac troponin $t$ measurement below the limit of detection: A collaborative meta-analysis. Ann Intern Med. 2017;166(10):715-724.

2. American Society for Clinical Pathology. Don't test for myoglobin or CK-MB in the diagnosis of acute myocardial infarction (AMI). Instead, use troponin I or T. http://www.choosingwisely.org/clinician-lists/american-society-clinical -pathology-myoglobin-to-diagnose-acute-myocardial-infarction/. Accessed August 3, 2016.

3. Amsterdam EA, Wenger NK, Brindis RG, et al. 2014 AHA/ACC guideline for the management of patients with non-st-elevation acute coronary syndromes. Circulation. 2014;130(25):e344-e426.

4. Larochelle MR, Knight AM, Pantle H, Riedel S, Trost JC. Reducing excess cardiac biomarker testing at an academic medical center. J Gen Intern Med. 2014;29(11):1468-1474.

5. Le RD, Kosowsky JM, Landman AB, Bixho I, Melanson SEF, Tanasijevic MJ.
Clinical and financial impact of removing creatine kinase-MB from the routine testing menu in the emergency setting. Am J Emerg Med. 2015;33(1):72-75.

6. Rosenberg A, Agiro A, Gottlieb M, et al. Early trends among seven recommendations from the choosing wisely campaign. JAMA Intern Med. 2015;175(12):1913.

7. Wolfson DB. Choosing Wisely recommendations using administrative claims data. JAMA Intern Med. 2016;176(4):565-565.

8. Thygesen K, Alpert JS, Jaffe AS, Simoons ML, Chaitman BR, White HD. Third universal definition of myocardial infarction. Circulation. 2012;126(16): 2020-2035.

9. US News \& World Report. Best hospitals for cardiology \& heart surgery. http:// health.usnews.com/best-hospitals/rankings/cardiology-and-heart-surgery. Accessed April 19, 2017.

10. Bradley EH, Curry LA, Ramanadhan S, Rowe L, Nembhard IM, Krumholz HM. Research in action: using positive deviance to improve quality of health care. Implement Sci IS. 2009;4:25. 